Our Nature (2010) 8:72-81

\title{
Birds of Natural Wetlands of North-west Punjab, India
}

\author{
R. Bal* and A. Dua \\ Aquatic Biology Lab., Department of Zoology, Guru Nanak Dev University, Amritsar-143001, Punjab, India \\ *E-mail: rupalibal@gmail.com
}

Received: 06.03.2010, Accepted: 19.08.2010

\begin{abstract}
Bird community of four natural wetlands around Gurdaspur, Punjab, India has been studied from April 2006-March 2010. Though various conservation strategies are implemented in protected areas still network of unrecognized natural wetlands are ignored. The network of such natural sites is important biodiversity corridor that facilitates the movement of migratory birds. The present study is step towards the protection of these valuable habitats, namely Keshopur Miani Community Reserve, Magarmudian chammb, Shalla Pattan wetland and Kahnuwan Wetland.
\end{abstract}

Key words: wetlands, birds, Punjab

\section{Introduction}

The recent studies assess freshwater biodiversity as the most threatened of all types of biodiversity and wetlands are found to be the richest sites by holding major share of the existing figure (Anon, 2000). It is estimated that freshwater wetlands alone support $20 \%$ of the known range of biodiversity in India (Deepa and Ramachandra, 1999).

Wetlands in India cover an area of 58.2 million hectares (Prasad et al., 2002). In Wildlife Institute of India's survey reveals that wetlands are disappearing at a rate of $2 \%$ to $3 \%$ every year. Freshwater ecosystems are key component in food web and play an important role in nutrient recycling. Many communities depend on the freshwater ecosystems for food and livelihood source. Wetlands are extremely essential to the continued existence of Indiaís diverse populations of wildlife and plant species; a large number of endemic species are wetland dependent. The fate of important birds like Sarus crane (VN) and
Common crane in India is dependent on the small natural wetlands. Natural wetlands of Gurdaspur now came into limelight as the most threatened crane population is coming regularly in winters. Bird diversity at Keshopur community reserve and Magarmudian is enormous. These four small wetlands host about 50 species of migratory birds and about 50000 migratory birds that flock the area during winter migratory season. These rich natural wetlands are extremely threatened because these valuable sites are still unprotected and waiting for action before being vanished in above mentioned sites in district Gurdaspur, Punjab, India. Wakefulness about the significance of wetlands is now very well known to all and was globally recognized.

\section{Study area}

Study sites are situated around Gurdaspur from north-west to south-east in clock wise direction i.e., Magarmudian Chhamb in north-west $\left(32^{\circ} 05^{\prime} 30^{\prime \prime} \mathrm{N}\right.$ and $\left.75^{\circ} 22^{\prime} 35^{\prime \prime} \mathrm{E}\right)$, 
Keshopur Miani Community Reserve in north $\left(32^{\circ} 05^{\prime} 47^{\prime \prime} \mathrm{N}\right.$ and $\left.75^{\circ} 24^{\prime} 34^{\prime \prime} \mathrm{E}\right)$, Shalla Pattan Wetland in east $\left(32^{\circ} 00^{\prime} 1.6^{\prime \prime} \mathrm{N}\right.$ and $\left.75^{\circ} 29^{\prime} 42^{\prime \prime} \mathrm{E}\right)$ and Kahnuwan Chhamb in south-east $\left(31^{\circ} 57^{\prime} 12^{\prime \prime} \mathrm{N}\right.$ and $\left.75^{\circ} 27^{\prime} 24^{\prime \prime} \mathrm{E}\right)$ (Figure 1). The above-mentioned wetlands are flood plain areas of the river Beas (Tributary of Indus river system). These wetlands are neither Ramsar sites nor do they find a place in National wetland map in India though qualifies and deserve it. They are at the verge of extinction and disintegration due to public apathy. Although these sites are dramatically altered by human activity still continues to provide habitat for many bird and animal species.

\section{Methodology}

Observations were made regularly at 15 days interval from April, 2006 to March, 2010 during most active period for birds. Co-ordinates of study sites were recorded with Garmin Global Positioning System. Direct count method was followed to take field data. 10-15 minutes were spent at each point to avoid repeated counting of same bird individual. Observations were carried out with the aid of $10-12 \times 50$ Nikon binoculars and photography was done with EOS 1000 D Canon with 75-300 mm telescopic lens. Identification of birds was done using field guides (Ali and Ripely, 1987; Grimmet et al., 1999). Abundance and status of recorded birds was established on frequency of occurrence during field visits. Status of species was classified into resident (R) and winter migrant (WM).

\section{Results and discussion}

A total of 233 species of birds belonging to 50 families were recorded from four small natural wetlands around Gurdaspur within
$10 \mathrm{~km}$ range Table 1 . Out of 233 recorded species, 72 are resident, 158 are migratory and 3 having sporadic occurrence. Highest number of birds was recorded in the month of January and lowest number was recorded in the month of June. Among four selected study sites two namely Keshopur Miani Community Reserve and Magarmudian chammb are holding major share as open water and other two namely Shalla Pattan and Kahnuwan are typically mashy lands along river Beas. Habitat preference has been observed in different bird species as common coot (Fulica atra), common poachard (Aythya ferina), pintail (Anas acuta), gadwall (Anas strepera), mallard (Anas platyrhynchos) dominates open water wetlands whereas common cranes (Grus grus), Sarus crane (Grus antigone), Whooly necked stork (Ciconia episcopus), Bar headed geese (Anser indicus) and huge variety of raptor dominates marshy lands. Our four year study establishes the richness of these wetlands in respect of bird fauna which are excellent indicators of ecological health. These wetlands are not Ramsar sites nor did they find any place in National wetland map though they qualifies and deserve it.

\section{References}

Ali, S. and S.D. Ripley 1987. Compact handbook of the birds of India and Pakistan together with those of Bangladesh, Nepal, Bhutan and Sri Lanka. Oxford University Press, Delhi.

Anonymous 2000. Wetland values and functions. The Ramsar Bureau. Gland, Swittzerland. pp. 2025.

Deepa, R.S. and T.V. Ramachandra 1999. Impact of urbanization in the interconnectivity of wetlands. Paper presented at the National Symposium on Remote Sensing Applications for Natural Resources: Retrospective and 


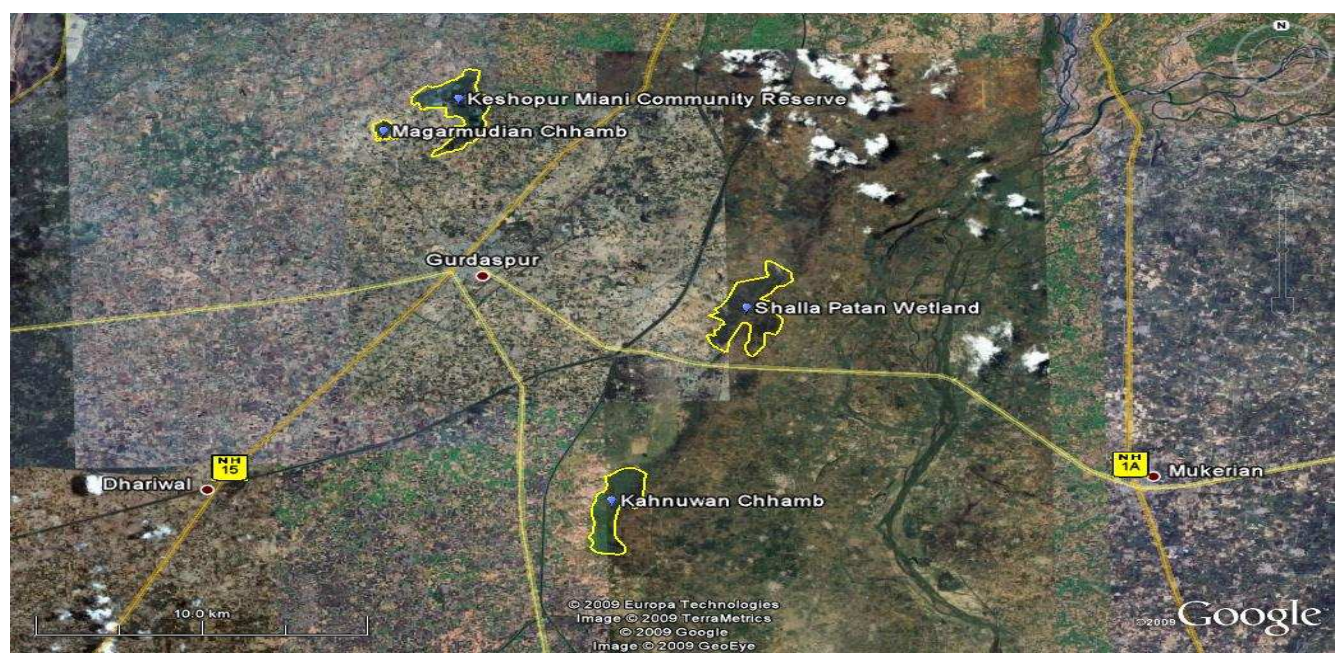

Figure 1. Map of the study area

Table 1. Total bird species recorded

\begin{tabular}{|c|c|c|}
\hline Common name & Scientific name & $\begin{array}{l}\text { Status / } \\
\text { occurrence }\end{array}$ \\
\hline \multicolumn{3}{|l|}{ Family: Accipitridae } \\
\hline Black Eagle & Ictinaetus malayensis & $\mathrm{IB} / \mathrm{R}$ \\
\hline Black Kite & Milvus migrans migrans & $\mathrm{RB} / \mathrm{UC}$ \\
\hline Black Winged Kite & Elanus caeruleus & $\mathrm{RB} / \mathrm{C}$ \\
\hline Bonellis Eagle & Hieraaetus fasciatus & $\mathrm{RB} / \mathrm{UC}$ \\
\hline Booted Hawk Eagle & Hieraaetus pennatus & $\mathrm{RB} / \mathrm{UC}$ \\
\hline Brahminy Kite & Haliastur indus & IB/UC \\
\hline Changeable Hawk Eagle & Spizaetus cirrhatus limnaeetus & $\mathrm{RB} / \mathrm{UC}$ \\
\hline Crested Hawk-Eagle & Spizaetus cirrhatus cirrhatus & $\mathrm{RB} / \mathrm{C}$ \\
\hline Crested Serpent Eagle & Spilornis cheela & $\mathrm{RB} / \mathrm{C}$ \\
\hline Great Spotted Eagle & Aquila clanga & $\mathrm{WM} / \mathrm{R}$ \\
\hline Hen Harrier & Circus cyaneus & $\mathrm{WM} / \mathrm{R}$ \\
\hline Honey Buzzard & Pernis ptilorhyncus & IB/UC \\
\hline Indian White Backed Vulture & Gyps bengalensis & $\mathrm{RB} / \mathrm{C}$ \\
\hline Lesser Spotted Eagle & Aquila pomarina & $\mathrm{WM} / \mathrm{R}$ \\
\hline Marsh Harrier & Circus aeruginosus & $\mathrm{WM} / \mathrm{C}$ \\
\hline Osprey & Pandion Haliaetus & $\mathrm{RB} / \mathrm{UC}$ \\
\hline Pallass Fishing Eagle & Haliaeetus leucoryphus & $\mathrm{RB} / \mathrm{UC}$ \\
\hline Pallid Harrier & Circus macrourus & $\mathrm{WM} / \mathrm{R}$ \\
\hline Pariah Kite & Milvus migrans govinda & IB/UC \\
\hline Scavenger Vulture & Neophron percnopterus & $\mathrm{RB} / \mathrm{C}$ \\
\hline Shikra & Accipiter badius & $\mathrm{RB} / \mathrm{UC}$ \\
\hline
\end{tabular}


R. Bal and A. Dua / Our Nature (2010) 8: 72-81

Short Toed Eagle

Circaetus gallicus

$\mathrm{RB} / \mathrm{UC}$

Tawny Eagle

Aquila rapax vindhiana

$\mathrm{WM} / \mathrm{R}$

White Eyed Buzzard

Butastur teesa

$\mathrm{RB} / \mathrm{R}$

\begin{tabular}{|c|c|c|}
\hline \multicolumn{3}{|l|}{ Family: Alaudidae } \\
\hline Ashy Crowned Sparrow Lark & Eremopterix grisea & $\mathrm{RB} / \mathrm{UC}$ \\
\hline Bush Lark & Mirafra assamica & $\mathrm{RB} / \mathrm{UC}$ \\
\hline Crested Lark & Galerida cristata & $\mathrm{RB} / \mathrm{C}$ \\
\hline Eastern Sky Lark & Alauda gulgula & $\mathrm{RB} / \mathrm{UC}$ \\
\hline Indian Bush Lark & Mirafra erythroptera & $\mathrm{RB} / \mathrm{UC}$ \\
\hline Sky Lark & Alauda arvensis & $\mathrm{RB} / \mathrm{UC}$ \\
\hline \multicolumn{3}{|l|}{ Family: Alcedinidae } \\
\hline Common Kingfisher & Alcedo atthis & $\mathrm{RB} / \mathrm{C}$ \\
\hline Himalayan Pied Kingfisher & Ceryle rudis & $\mathrm{WM} / \mathrm{C}$ \\
\hline Lesser Pied Kingfisher & Megaceryle lugubris & $\mathrm{WM} / \mathrm{R}$ \\
\hline Stork Billed Kingfisher & Pelargopsis capensis & $\mathrm{RB} / \mathrm{UC}$ \\
\hline White-Breasted Kingfisher & Halcyon smyrnensis & $\mathrm{RB} / \mathrm{C}$ \\
\hline \multicolumn{3}{|l|}{ Family: Anatidae } \\
\hline Common Merganser & Mergus merganser & $\mathrm{WM} / \mathrm{R}$ \\
\hline Common Pochard & Aythya ferina & $\mathrm{WM} / \mathrm{C}$ \\
\hline Common Teal & Anas crecca & $\mathrm{WM} / \mathrm{C}$ \\
\hline Eurasian Wigeon & Anas penelope & $\mathrm{WM} / \mathrm{C}$ \\
\hline Gadwall & Anas strepera & $\mathrm{WM} / \mathrm{C}$ \\
\hline Garganey & Anasquer quedula & WM/UC \\
\hline Lesser Whistling Duck & Dendrocygna javanica & $\mathrm{RB} / \mathrm{C}$ \\
\hline Mallard & Anas platyrhynchos & $\mathrm{WM} / \mathrm{UC}$ \\
\hline Pintail & Anas acuta & $\mathrm{WM} / \mathrm{C}$ \\
\hline Redcrested Pochard & Netta rufinna & $\mathrm{WM} / \mathrm{C}$ \\
\hline Ruddy Shelduck(Brahminy Duck) & Tadorna ferruginea & $\mathrm{WM} / \mathrm{C}$ \\
\hline Shoveler & Anas clypeata & $\mathrm{WM} / \mathrm{C}$ \\
\hline Greylag Geese & Anser anser & $\mathrm{WM} / \mathrm{C}$ \\
\hline Barheaded Geese(Nanjal) & Anser indicus & $\mathrm{WM} / \mathrm{C}$ \\
\hline \multicolumn{3}{|l|}{ Family: Apodidae } \\
\hline House Swift & Apus affinis & $\mathrm{RB} / \mathrm{C}$ \\
\hline Palm Swift & Cypsiurus balasiensis & $\mathrm{RB} / \mathrm{UC}$ \\
\hline \multicolumn{3}{|l|}{ Family: Ardeidae } \\
\hline Black Bittern & Ixobrychus flavicollis & $\mathrm{RB} / \mathrm{UC}$ \\
\hline Cattle Egret & Bubulcus ibis & $\mathrm{RB} / \mathrm{C}$ \\
\hline Cinnamon Bittern & Ixobrychus cinnamomeus & $\mathrm{RB} / \mathrm{UC}$ \\
\hline Grey Heron & Ardea cinerea & $\mathrm{RB} / \mathrm{UC}$ \\
\hline Large Egret & Ardea alba & $\mathrm{RB} / \mathrm{C}$ \\
\hline Little Egret & Egretta garzetta & $\mathrm{RB} / \mathrm{C}$ \\
\hline Night Heron & Nycticorax nycticorax & $\mathrm{RB} / \mathrm{UC}$ \\
\hline
\end{tabular}


R. Bal and A. Dua / Our Nature (2010) 8: 72-81

\begin{tabular}{|c|c|c|}
\hline Pond Heron Or Paddy Bird & Ardeola grayii & $\mathrm{RB} / \mathrm{C}$ \\
\hline Purple Heron & Ardea purpurea & $\mathrm{RB} / \mathrm{C}$ \\
\hline Smaller Egret & Egretta intermedia & $\mathrm{RB} / \mathrm{C}$ \\
\hline Yellow Bittern & Ixobrychus sinensis & RB/UC \\
\hline \multicolumn{3}{|l|}{ Family: Bucerotidae } \\
\hline Common Grey Hornbill & Ocyceros birostris & $\mathrm{RB} / \mathrm{C}$ \\
\hline Indian Pied Hornbill & Anthracoceros albiroslris & $\mathrm{RB} / \mathrm{C}$ \\
\hline \multicolumn{3}{|l|}{ Family: Campephagidae } \\
\hline Bay Backed Shrike & Lanius viltatus & $\mathrm{RB} / \mathrm{C}$ \\
\hline Long Tailed Shrike & Lanius schach & $\mathrm{RB} / \mathrm{C}$ \\
\hline Scarlet Minivet & Pericrocotus flammeus & WM/UC \\
\hline Small Minivet & Pericrocotus cinnamomeus & $\mathrm{RB} / \mathrm{C}$ \\
\hline \multicolumn{3}{|l|}{ Family: Capitonidae } \\
\hline Blue Throated Barbet & Megalaima asiatica & RB/UC \\
\hline Coppersmith Barbet & Megalaima haemacephala & $\mathrm{IB} / \mathrm{UC}$ \\
\hline Large Green Barbet & Megalaima zeylanica & $\mathrm{RB} / \mathrm{C}$ \\
\hline \multicolumn{3}{|l|}{ Family: Caprimulgidae } \\
\hline Franklin,S Nightjar & Caprimulgusaffinis & $\mathrm{RB} / \mathrm{C}$ \\
\hline Little Nightjar & Caprimulgus asiaticus & RB/UC \\
\hline \multicolumn{3}{|l|}{ Family: Caruidae } \\
\hline Common Crane & Grus grus & $\mathrm{WM} / \mathrm{C}$ \\
\hline Demoiselle Crane & Grus virgo & $\mathrm{WM} / \mathrm{R}$ \\
\hline Sarus Crane & Grus antigone & $\mathrm{RB} / \mathrm{C}$ \\
\hline \multicolumn{3}{|l|}{ Family: Charadriidae } \\
\hline Bartailed Godwit & Limo'sa lapponica & $\mathrm{WM} / \mathrm{R}$ \\
\hline Black-Tailed Godwit & Limosa limosa & $\mathrm{WM} / \mathrm{R}$ \\
\hline Common Redshank & Tringa totanus & $\mathrm{WM} / \mathrm{C}$ \\
\hline Common Sandpiper & Tringa hypoleucos & $\mathrm{WM} / \mathrm{C}$ \\
\hline Curlew (Eurasian) & Numenius arquata & $\mathrm{WM} / \mathrm{R}$ \\
\hline Fantail Snip (Common) & Gallinago allinago & WM/UC \\
\hline Green Sandpiper & Tringa ochropus & WM/C \\
\hline Green Shank & Tringa nebularia & $\mathrm{WM} / \mathrm{C}$ \\
\hline Jack Snipe & Gallinago minima & WM/UC \\
\hline Kentish Plover & Charadrius alexandrinus & WM/UC \\
\hline Little Ringed Plover & Charadrius dubius & $\mathrm{BR} / \mathrm{C}$ \\
\hline Little Stint & Calidris minuta & $\mathrm{WM} / \mathrm{C}$ \\
\hline Long-Toed Stint & Calidris subminuta & $\mathrm{WM} / \mathrm{C}$ \\
\hline Marsh Sandpiper & Tringa stagnatilis & $\mathrm{WM} / \mathrm{C}$ \\
\hline Northern Lapwing & Vanellus vanellus & WM/UC \\
\hline Red-Wattled Lapwing & Vanallus indicus & $\mathrm{RB} / \mathrm{C}$ \\
\hline Ruff And Reave & Philomachus pugnax & $\mathrm{WM} / \mathrm{C}$ \\
\hline Sppoted Or Dusky Redshank & Tringa erythropus & $\mathrm{WM} / \mathrm{R}$ \\
\hline
\end{tabular}


R. Bal and A. Dua / Our Nature (2010) 8: 72-81

\begin{tabular}{|c|c|c|}
\hline White Tailed Lapwing & Vanellus leucurus & WM/UC \\
\hline Wood Sandpiper & Tringa glareola & WM/UC \\
\hline Yellow-Wattled Lapwing & Vanellus malabaricus & $\mathrm{IB} / \mathrm{R}$ \\
\hline \multicolumn{3}{|l|}{ Family: Ciconidae } \\
\hline Painted Stork, & My cteria leuco cephala & RB/UC \\
\hline Woolly Necked Stork & Ciconia episcopus & $\mathrm{WM} / \mathrm{C}$ \\
\hline \multicolumn{3}{|l|}{ Family: Columbidae } \\
\hline Blue Rock Pigeon & Columba livia & $\mathrm{RB} / \mathrm{C}$ \\
\hline Indian Ring Dove & Steptopelia decaocto & $\mathrm{RB} / \mathrm{C}$ \\
\hline Little Brown Dove & Streptopelia senegalensis & $\mathrm{RB} / \mathrm{C}$ \\
\hline Red Turtle Dove & Sterptopelia tranquebarica & $\mathrm{RB} / \mathrm{C}$ \\
\hline Rufous Turtule Dove & Streptopelia orientalis & RB/UC \\
\hline Spotted Dove & Sterptopelia chinensis & $\mathrm{RB} / \mathrm{C}$ \\
\hline \multicolumn{3}{|l|}{ Family: Coraciidae } \\
\hline Indian Roller & Coracias benghalensis & $\mathrm{RB} / \mathrm{C}$ \\
\hline \multicolumn{3}{|l|}{ Family: Corvidae } \\
\hline House Crow & Corvus splendens & $\mathrm{RB} / \mathrm{C}$ \\
\hline Indain Tree Pie (Rufous) & Dendrocitta vagabunda & $\mathrm{RB} / \mathrm{C}$ \\
\hline Jungle Crow & Corvus macrorhynchos & $\mathrm{RB} / \mathrm{C}$ \\
\hline \multicolumn{3}{|l|}{ Family: Cuculidae } \\
\hline Common Hawk Cuckoo & Cuculus varius & $\mathrm{SM} / \mathrm{C}$ \\
\hline Indian Cuckoo & Cuculus micropterus & $\mathrm{SM} / \mathrm{C}$ \\
\hline Koel-Cuckoo & Eudynamys scolopacea & $\mathrm{RB} / \mathrm{C}$ \\
\hline Large Couca (Crow Pheasant) & Centropus sinensis & $\mathrm{RB} / \mathrm{C}$ \\
\hline Lesser Coucal & Centropus toulou & $\mathrm{RB} / \mathrm{C}$ \\
\hline The Cuckoo & Cuculus canorus & SM/UC \\
\hline \multicolumn{3}{|l|}{ Family: Dicruridae } \\
\hline Ashy Drongo & Dicrurus Ieucophaeus & $\mathrm{RB} / \mathrm{C}$ \\
\hline Black Drongo & Dicrurus adsimilis & $\mathrm{RB} / \mathrm{C}$ \\
\hline White-Bellied Drongo & Dicrurus caerulescens & $\mathrm{RB} / \mathrm{C}$ \\
\hline \multicolumn{3}{|l|}{ Family: Falconidae } \\
\hline Laggar Falcon & Falco biarmicus jugger & $\mathrm{WM} / \mathrm{R}$ \\
\hline Peregrine Falcon & Falco peregrinus japonensis & $\mathrm{WM} / \mathrm{R}$ \\
\hline \multicolumn{3}{|l|}{ Family: Hirundinidae } \\
\hline Plain Sand Martin & Riparia paludicola & RB/UC \\
\hline Swallow & Hirundo rustica & WM/UC \\
\hline Wire-Tailed Swallow & Hirundo smithii & $\mathrm{RB} / \mathrm{UC}$ \\
\hline \multicolumn{3}{|l|}{ Family: Irenidae } \\
\hline Iora (Indian Iora) & Aegithina tiphia & RB/UC \\
\hline \multicolumn{3}{|l|}{ Family: Jacanidae } \\
\hline Bronzewinged Jacana & Metopidius indicus & $\mathrm{RB} / \mathrm{C}$ \\
\hline Pheasant Tailed Jacana & Hydrophasianus chirurgus & $\mathrm{RB} / \mathrm{C}$ \\
\hline
\end{tabular}


R. Bal and A. Dua / Our Nature (2010) 8: 72-81

\begin{tabular}{|c|c|c|}
\hline \multicolumn{3}{|l|}{ Family: Laniidae } \\
\hline Bay Backed Shrike & Lanius vittatus & $\mathrm{RB} / \mathrm{UC}$ \\
\hline Blacked Shrike Or Rufous (Longtailed) & Lanius schach & $\mathrm{IB} / \mathrm{R}$ \\
\hline Southern Grey Shrike & Lanius meridionalis & $\mathrm{RB} / \mathrm{UC}$ \\
\hline \multicolumn{3}{|l|}{ Family: Laridae } \\
\hline Black Bellied Tern & Sterna acuticauda & $\mathrm{IB} / \mathrm{R}$ \\
\hline Black Headed Gull & Larus ridibundus & $\mathrm{WM} / \mathrm{R}$ \\
\hline Brown Headed Gull & Larus brunnicephalus & $\mathrm{WM} / \mathrm{R}$ \\
\hline Common Tern & Sterna hirundo & $\mathrm{RB} / \mathrm{C}$ \\
\hline Great Black Headed Gull & Larus ichthyaetus & $\mathrm{WM} / \mathrm{R}$ \\
\hline Indian River Tern & Sterna aurantia & $\mathrm{RB} / \mathrm{C}$ \\
\hline \multicolumn{3}{|l|}{ Family: Meropidae } \\
\hline Blue-Tailed Bee-Eater & Merops philippinus & $\mathrm{RB} / \mathrm{C}$ \\
\hline Green Bee-Eater & Merops orientalis & $\mathrm{RB} / \mathrm{C}$ \\
\hline \multicolumn{3}{|l|}{ Family: Motacillidae } \\
\hline Grey-Wagtail & Motacilla cinerea & $\mathrm{WM} / \mathrm{C}$ \\
\hline Pied Wagtail (White Wagtail) & Motacilla alba & $\mathrm{WM} / \mathrm{C}$ \\
\hline Yellow Headed Wagtail/ Citrine Wagtail & Motacilla citreola & $\mathrm{WM} / \mathrm{C}$ \\
\hline Yellow Wagtail & Motacilla flava & $\mathrm{WM} / \mathrm{C}$ \\
\hline \multicolumn{3}{|l|}{ Family: Muscicapinae } \\
\hline Blue-Throated Fly Catcher & Muscicapa rubeculoides & IB/UC \\
\hline Darksided Flycatcher & Muscicapa sibirica & WM/UC \\
\hline Rufous Gorgeted Flycatcher & Ficedula strophiata & RB/UC \\
\hline Slaty Blue Fly Catcher & Ficedula tricolor & WM/UC \\
\hline Tickell'S Blue Fly Catcher & Muscicapa tickelliae & $\mathrm{RB} / \mathrm{UC}$ \\
\hline White-Browed Fan Tail Fly Catcher & Rhipidura aureola & RB/UC \\
\hline White-Throated Fantail Fly Catcher & Rhipidura albicollis & $\mathrm{RB} / \mathrm{UC}$ \\
\hline Yellow Bellied Fan Tail Fly Catcher & Rhipidura hypoxantha & WM/UC \\
\hline Red-Breasted Fly Catcher & Ficedula strophiata parya & $\mathrm{WM} / \mathrm{C}$ \\
\hline \multicolumn{3}{|l|}{ Family: Nectariniidae } \\
\hline Crimson Sunbird & Aethopyga siparaja & $\mathrm{RB} / \mathrm{UC}$ \\
\hline Purple Sun Bird & Nectarinia asiatica & $\mathrm{RB} / \mathrm{C}$ \\
\hline \multicolumn{3}{|l|}{ Family:ORIOLIDAE } \\
\hline Black Headed Oriole & Oriolous xanthornus & $\mathrm{RB} / \mathrm{C}$ \\
\hline Black Naped Oriole & Oriolous chinensis & $\mathrm{RB} / \mathrm{UC}$ \\
\hline Eurasian Golden Oriole & Oriolous oriolus & $\mathrm{RB} / \mathrm{UC}$ \\
\hline \multicolumn{3}{|l|}{ Family: Pachycephalinae, Sub- Sylviinae } \\
\hline Brown Leaf Warbler Or Chiffchaff & Phylloscopus collybita & WM/UC \\
\hline Dull Green Leaf Warbler & Phylloscopus trochiloides & WM/UC \\
\hline Striated Marsh Warbler & Megalurus palustris & $\mathrm{RB} / \mathrm{UC}$ \\
\hline Tailor Bird & Orthotomus sutorius & $\mathrm{RB} / \mathrm{C}$ \\
\hline
\end{tabular}

Family: Pachycephalinae, Sub- Turdinae 
R. Bal and A. Dua / Our Nature (2010) 8: 72-81

\begin{tabular}{|c|c|c|}
\hline Black Redstart & Phoenicurus ochruros & WM/UC \\
\hline Brown Rock Chat & Cercomela fusca & $\mathrm{RB} / \mathrm{UC}$ \\
\hline Collared Bush Chat (Stone Chat) & Saxicola torquata & $\mathrm{RB} / \mathrm{C}$ \\
\hline Dark-Grey Bush Chat & Saxicola ferrea & WM/UC \\
\hline Magpie Robin & Copsychus saularis & $\mathrm{RB} / \mathrm{C}$ \\
\hline Pied Bush Chat & Saxicola caprata & $\mathrm{RB} / \mathrm{C}$ \\
\hline White Capped Water Redstart & Chaimarrornis leucocephalus & $\mathrm{RB} / \mathrm{C}$ \\
\hline White Tailed Stone Chat & Saxicola leucura & IB/UC \\
\hline \multicolumn{3}{|l|}{ Family: Paridae } \\
\hline Black Lored Tit & Parus xanthogenys & $\mathrm{RB} / \mathrm{R}$ \\
\hline Great Tit & Parus major & $\mathrm{RB} / \mathrm{C}$ \\
\hline \multicolumn{3}{|l|}{ Family: Phalacrocoracidae } \\
\hline Darter & Anhinga rufa & $\mathrm{RB} / \mathrm{C}$ \\
\hline Indian Cormorant & Phalacrocorax fuscicollis & $\mathrm{IB} / \mathrm{C}$ \\
\hline Large Cormorant & Phalacrocorax carbo & $\mathrm{RB} / \mathrm{C}$ \\
\hline Little Cormorant & Phalacrocorax niger & $\mathrm{RB} / \mathrm{C}$ \\
\hline \multicolumn{3}{|l|}{ Family: Phas ianidae } \\
\hline Black Breasted Quail (Rain Quail) & Coturnix coromandelica & $\mathrm{RB} / \mathrm{C}$ \\
\hline Black Partridges/Francolin & Francolinus francolinus & $\mathrm{RB} / \mathrm{C}$ \\
\hline Blue Breasted Quail & Coturnix chinensis & $\mathrm{RB} / \mathrm{C}$ \\
\hline Common Peafowl & Pavo cristatus & $\mathrm{RB} / \mathrm{C}$ \\
\hline Grey Partridges /Francolin & Francolinus pondicerianus & $\mathrm{RB} / \mathrm{C}$ \\
\hline Grey Quail(Common) & Coturnix coturnix & $\mathrm{RB} / \mathrm{C}$ \\
\hline Red Jungle Fowl & Gallus gallus & $\mathrm{RB} / \mathrm{C}$ \\
\hline \multicolumn{3}{|l|}{ Family: Picidae } \\
\hline Black Backed Woodpecker & Chrysocoleptes fectivus & $\mathrm{RB} / \mathrm{R}$ \\
\hline Golden Becked Woodpecker & Dinopium shorii & $\mathrm{RB} / \mathrm{C}$ \\
\hline Himalayan Pied Woodpecker & Dendrocopos himalaYensis & $\mathrm{RB} / \mathrm{UC}$ \\
\hline Larger Golden Backed Woodpecker & Chrysocolaptes lucidus & IB/UC \\
\hline $\begin{array}{l}\text { Lesser Golden Backed Woodpecker/ Black } \\
\text { Fumped Flameback }\end{array}$ & Dinopium benghalense & $\mathrm{RB} / \mathrm{C}$ \\
\hline Wryneck & Jynx torquilla & WM/UC \\
\hline Yellow Crowned Woodpecker & Dendrocopos mahrattensis & $\mathrm{RB} / \mathrm{UC}$ \\
\hline \multicolumn{3}{|l|}{ Family: Pittidae } \\
\hline Indian Pitta & Pitta brachyura & $\mathrm{RB} / \mathrm{C}$ \\
\hline \multicolumn{3}{|l|}{ Family: Ploceidae } \\
\hline Baya, Weaver Birds & Ploceus philippinus & $\mathrm{RB} / \mathrm{C}$ \\
\hline Black Throated Weaver Bird / Black Breasted & Ploceus benghalensis & RB/UC \\
\hline Chest Nut- Shouldered Petronia & Petronia xanthocollis & $\mathrm{RB} / \mathrm{C}$ \\
\hline House Sparrow & Passer domesticus & $\mathrm{RB} / \mathrm{C}$ \\
\hline Red Munia Or Avadavat & $\begin{array}{l}\text { Estrilda amandava / Amandava } \\
\text { amandava }\end{array}$ & $\mathrm{RB} / \mathrm{C}$ \\
\hline Scaly-Breasted Munia/Spotted Munia & Lonchura punctulata & $\mathrm{RB} / \mathrm{C}$ \\
\hline
\end{tabular}


R. Bal and A. Dua / Our Nature (2010) 8: 72-81

\begin{tabular}{|c|c|c|}
\hline Streaked Weaver Bird & Ploceus manyar & RB/UC \\
\hline Spanish Sparrow & Passer hispaniolensis & WM/UC \\
\hline \multicolumn{3}{|l|}{ Family: Podicipedidea } \\
\hline Great Crested Grebe & Podiceps cristatus & WM/R \\
\hline Little Grebe & Podiceps ruficollis & $\mathrm{RB} / \mathrm{UC}$ \\
\hline \multicolumn{3}{|l|}{ Family: Prionopidae } \\
\hline Common Wood Shrike & Tephrodornis pondicerianus & $\mathrm{RB} / \mathrm{C}$ \\
\hline \multicolumn{3}{|l|}{ Family: Psittacidae } \\
\hline Alexandrine Parakeet & Psittacula eupatria & $\mathrm{RB} / \mathrm{C}$ \\
\hline Grey Headed Parakeet & Psittacula finschii & $\mathrm{RB} / \mathrm{C}$ \\
\hline Plum Headed Parakeet & Psittacula cyanocephala & $\mathrm{RB} / \mathrm{C}$ \\
\hline Rose Ringed Parakeet & Psittacula kramei & $\mathrm{RB} / \mathrm{C}$ \\
\hline \multicolumn{3}{|l|}{ Family: Pycnonotidae } \\
\hline Black-Headed Yellow Bulbul & Pycnonotus melanicterus flaviventris & $\mathrm{RB} / \mathrm{UC}$ \\
\hline Grey Bulbul (Black Bulbul) & Hypsipetes madagascariensis & $\mathrm{RB} / \mathrm{C}$ \\
\hline Red Vented Bulbul & Pycnonotus cafer & $\mathrm{RB} / \mathrm{C}$ \\
\hline Red Whiskered Bulbul & Pycnonotus jocosus & $\mathrm{RB} / \mathrm{C}$ \\
\hline White Cheeked Bulbul & Pycnonotus leucogenys & $\mathrm{RB} / \mathrm{UC}$ \\
\hline \multicolumn{3}{|l|}{ Family: Rallidae } \\
\hline Coot & Fulica atra & WM/C \\
\hline Indian Gallinule/Indian Moorhen & Gcrllinula chloropus & $\mathrm{RB} / \mathrm{C}$ \\
\hline Purple Gallinule/Purple Moorhen & Porphyrio porphyrio & $\mathrm{RB} / \mathrm{C}$ \\
\hline Water Cock & Gallicrex cincrea & $\mathrm{W} / \mathrm{R}$ \\
\hline White Breasted Water Hen & Amaurornis phoenicurus & $\mathrm{RB} / \mathrm{C}$ \\
\hline \multicolumn{3}{|l|}{ Family: Recurvirostridae } \\
\hline Avocet & Recurvirostra avosetta & $\mathrm{WM} / \mathrm{UC}$ \\
\hline Black Winged Stilt & Himantopus himantonus & $\mathrm{RB} / \mathrm{C}$ \\
\hline \multicolumn{3}{|l|}{ Family: Strigidae } \\
\hline Barn Owl & Tyto alba & $\mathrm{RB} / \mathrm{R}$ \\
\hline Barred Owlet & Glaucidium cuculoides & RB/UC \\
\hline Great Horned Owl (Eagle Owl) & Bubo bubo & $\mathrm{RB} / \mathrm{UC}$ \\
\hline Jungle Owlet & Glaucidium radiatum & $\mathrm{RB} / \mathrm{C}$ \\
\hline Spotted Owlet & Athene brama & $\mathrm{RB} / \mathrm{UC}$ \\
\hline \multicolumn{3}{|l|}{ Family: Sturnidae } \\
\hline Bank Myna & Acridotheres ginginianus & $\mathrm{RB} / \mathrm{C}$ \\
\hline Common Myna & Acridotheres tristis & $\mathrm{RB} / \mathrm{C}$ \\
\hline Common Starling & Sturnus vulgaris & $\mathrm{WM} / \mathrm{C}$ \\
\hline Jungle Myna & Acridotheres fuscus & $\mathrm{RB} / \mathrm{UC}$ \\
\hline \multicolumn{3}{|l|}{ Family: Tadorna } \\
\hline Common Shelduck & Tadorna tadorna & WM/UC \\
\hline \multicolumn{3}{|l|}{ Family: Threskiornithidae } \\
\hline Black Headed Ibis & Threskiornis melanocephalus & WM/C \\
\hline
\end{tabular}


R. Bal and A. Dua / Our Nature (2010) 8: 72-81

\begin{tabular}{lll} 
Black Ibis (Nanjal) & $\begin{array}{l}\text { Pseudibis papillosa } \\
\text { Platalea leucorodiu }\end{array}$ & $\mathrm{RB} / \mathrm{C}$ \\
Spoonbill (Nanjal) & WM/R \\
\hline Family: Timalinae & $\begin{array}{l}\text { Turdoides striatus } \\
\text { Jungle Babbler (Seven Sisters) }\end{array}$ & Turdoides earlei \\
Striated Babbler & Garrulax albogularis : & $\mathrm{RB} / \mathrm{C}$ \\
White Throated Laughing-Thrush & Turnix suscitator & $\mathrm{WM} / \mathrm{R}$ \\
\hline Family: Turnicidae & & $\mathrm{RB} / \mathrm{R}$ \\
Barred Button Quail & Upupa epops & $\mathrm{RB} / \mathrm{C}$ \\
\hline Family: Upupidae & Hoopoe &
\end{tabular}

$\mathrm{RB}=$ Resident breeders, $\mathrm{S}=$ Sporadic, $\mathrm{WM}=$ Winter migrant, $\mathrm{UC}=$ Uncommon, $\mathrm{R}=\mathrm{Rare}, \mathrm{C}=\mathrm{Common}$

Perspective (XIX-XXI, 1999), Indian Society of Remote Sensing, Banglore.

Grimmett, R., C. Inskipp and T. Inskipp 1999. Pocket guide to the birds of the Indian subcontinent. Oxford University Press, Delhi.
Prasad, S.N., T.V. Ramachandra, N. Ahalya, T. Sengupta, A. Kumar, A.K. Tiwari, V.S. Vijayan and L. Vijayan 2002. Conservation of wetlands of India- A review. Tropical Ecology 43(1): 173-186. 